\title{
Nonlinear Interactions between Excitatory and Inhibitory Retinal Synapses Control Visual Output
}

\author{
Botir T. Sagdullaev, ${ }^{1}$ Erika D. Eggers, ${ }^{2}$ Robert Purgert, ${ }^{3}$ and Peter D. Lukasiewicz ${ }^{3}$ \\ ${ }^{1}$ Departments of Ophthalmology and Neurology, Weill Medical College of Cornell University, Burke Medical Research Institute, White Plains, New York \\ 10605, ${ }^{2}$ Departments of Physiology and Biomedical Engineering, University of Arizona, Tucson, Arizona 85724, and ${ }^{3}$ Department of Ophthalmology and \\ Visual Sciences, Washington University School of Medicine, St. Louis, Missouri 63110
}

The visual system is highly sensitive to dynamic features in the visual scene. However, it is not known how or where this enhanced sensitivity first occurs. We investigated this phenomenon by studying interactions between excitatory and inhibitory synapses in the second synaptic layer of the mouse retina. We found that these interactions showed activity-dependent changes that enhanced signaling of dynamic stimuli. Excitatory signaling from cone bipolar cells to ganglion cells exhibited strong synaptic depression, attributable to reduced glutamate release from bipolar cells. This depression was relieved by amacrine cell inhibitory feedback that activated presynaptic $\mathrm{GABA}_{\mathrm{C}}$ receptors. We found that the balance between excitation and feedback inhibition depended on stimulus frequency; at short interstimulus intervals, excitation was enhanced, attributable to reduced inhibitory feedback. This dynamic interplay may enrich visual processing by enhancing retinal responses to closely spaced temporal events, representing rapid changes in the visual environment.

\section{Introduction}

Natural visual scenes contain features that continuously change and features that remain static. Dynamic visual inputs result in complex stimulation of neural circuits. Transmission of dynamic signals differs from that of static signals because activity-dependent changes in synaptic efficacy shape temporally changing, but not static signals (Zucker and Regehr, 2002). This time-dependent gain control can be used to maintain reliable transmission over a range of stimulus conditions, while retaining the ability to respond to novel stimuli.

Synaptic depression, a short-term form of depression induced by repetitive stimulation, is present at most synapses and may have diverse effects on neuronal signaling. In some cases, synaptic depression reduces the reliability of synaptic transmission (Trussell et al., 1993; Brenowitz and Trussell, 2001; Chen et al., 2002), while in other cases it enhances the fidelity of transmission (Abbott et al., 1997). Synaptic depression has been attributed to both presynaptic and postsynaptic mechanisms. Presynaptically, repetitive stimulation can lead to reduced neurotransmitter release (del Castillo and Katz, 1954; Furukawa and Matsuura, 1978; Dittman and Regehr, 1998; Bellingham and Walmsley, 1999; Singer and Diamond, 2006). Postsynaptically, repetitive stimulation can produce receptor desensitiza-

\footnotetext{
Received April 11, 2011; revised Aug. 27, 2011; accepted Aug. 30, 2011.

Author contributions: B.T.S. and P.D.L. designed research; B.T.S., E.D.E., and R.P. performed research; B.T.S., E.D.E., and R.P. analyzed data; B.T.S. and P.D.L. wrote the paper.

This work was supported by NIH Grants EY08922 (P.D.L.), EY020535 (B.T.S.), EY018131 (E.D.E.), and EY02687 (Washington University Department of Ophthalmology), Research to Prevent Blindness, and The M. Bauer Foundation. We thank Christopher Yee for assistance in writing program routines; and Drs. Daniel Kerschensteiner, Steven Mennerick, and members of the Lukasiewicz laboratory for comments on this manuscript.

Correspondence should be addressed to either of the following: Peter D. Lukasiewicz at the above address, E-mail: Lukasiewicz@vision.wustl.edu; or Botir T. Sagdullaev at the above address, E-mail: bos2005@med. cornell.edu.

DOI:10.1523/JNEUROSCI.1801-11.2011

Copyright $\odot 2011$ the authors $\quad 0270-6474 / 11 / 3115102-11 \$ 15.00 / 0$
}

tion and/or saturation (Trussell and Fischbach, 1989; Trussell et al., 1993; Chen et al., 2002).

In the retina, synaptic depression contributes to neural adaptation in the rod-signaling pathway (Dunn and Rieke, 2008). This synaptic depression is attributed to reduced excitation and is not mediated by inhibition. Neural circuits in other parts of the CNS minimize synaptic depression to maintain reliable transmission, using presynaptic inhibition. At both the nucleus magnocellularis and retinogeniculate synapses, presynaptic inhibition lessens depression by reducing transmitter release (Brenowitz et al., 1998; Brenowitz and Trussell, 2001; Chen and Regehr, 2003; Cook et al., 2003). The role of synaptic depression in conesignaling pathways of the retina is unknown. It is also not known whether presynaptic inhibition modulates retinal synaptic depression (Demb and von Gersdorff, 2008). Does presynaptic inhibition reduce depression, enabling retinal synapses to respond to novel stimuli?

To investigate this, we studied a retinal circuit, composed of ON cone bipolar cells (BCs), amacrine cells, and ON-center ganglion cells (GCs), that responds to increments in illumination. In this circuit, excitatory $\mathrm{BC}$ output activates GABAergic amacrine cells that feed back via $G_{A B A}$ receptor $\left(G_{A B A} R\right)$-mediated presynaptic inhibition to modulate excitatory transmission from BCs to GCs (Matsui et al., 2001; Sagdullaev et al., 2006). We found that activation of presynaptic $\mathrm{GABA}_{\mathrm{C}} \mathrm{Rs}$ reduced depression of $\mathrm{BC}$ excitatory transmission by limiting transmitter release and preventing transmitter depletion. The effect of presynaptic inhibition depended on the frequency of the stimuli. As the interstimulus intervals (ISIs) shortened, presynaptic inhibition that normally reduced the second response progressively decreased. This dynamic interplay reduced synaptic depression, allowing the circuit to strongly respond to closely timed inputs. Ultimately, this interaction between excitation and inhibition at ret- 
inal reciprocal synapses enhances the ability of ON-center GCs to generate spikes in response to repetitive light stimulation and preserves specific temporal features of visual input.

\section{Materials and Methods}

In all experimental procedures, the animals were treated according to the regulations in The Association for Research in Vision and Ophthalmology Statement for the Use of Animals in Ophthalmic and Vision Research and in compliance with protocols approved by both Washington University and Weill Cornell Medical College. Wild-type (C57BL/6J) mice of either sex were obtained from The Jackson Laboratory.

Retina wholemount and slice preparations. Retinas were isolated as described previously (McCall et al., 2002). Eyes were enucleated and placed in oxygenated HEPES-buffered extracellular Ringer's solution 1, containing the following (in mM): $137 \mathrm{NaCl}, 2.5 \mathrm{KCl}, 2.5 \mathrm{CaCl}_{2}, 1.0 \mathrm{MgCl}_{2}$, 10 Na-HEPES, 28 glucose, pH 7.4. The cornea, iris, and lens were removed with small scissors. For the wholemount preparation, the retina was dissected into four equal pieces. Using gentle suction, each quadrant was attached, photoreceptor surface down, to a modified translucent Millipore Millicell filter ring. To prepare retinal slices, the isolated retina was placed on Millipore filter paper, vitreal side down, and slices were cut at $200-250 \mu \mathrm{m}$ intervals and stored in oxygenated extracellular solution 1. Individual slices were transferred to a recording chamber one at a time and mounted on the stage of an upright microscope equipped with Hoffman modulation contrast optics (Modulation Optics), as described previously (Lukasiewicz and Roeder, 1995). The recording chamber was constantly superfused ( $1 \mathrm{ml} / \mathrm{min}$ ) with bicarbonate-buffered extracellular Ringer's solution 2, containing the following (in mM): $125 \mathrm{NaCl}, 2.5$ $\mathrm{KCl}, 2.0 \mathrm{CaCl}_{2}, 1.0 \mathrm{MgCl}_{2}, 1.25 \mathrm{NaH}_{2} \mathrm{PO}_{4}, 26 \mathrm{NaHCO}_{3}$, and 20 glucose, which was bubbled with $95 \% \mathrm{O}_{2}$ and $5 \% \mathrm{CO}_{2}$. In slice experiments (unless otherwise specified), control extracellular solution 2 contained $100 \mu \mathrm{M}$ bicuculline and $5 \mu \mathrm{M}$ strychnine. To avoid any potential network effects of blocking inhibition, experiments in the wholemount retinas were performed in bicarbonate-buffered Ringer's solution. Pharmacological agents were bath applied in the control solution using a computer-controlled, multichannel superfusion system. Reagents including CNQX (6-cyano-7-nitroquinoxaline-2,3-dione) and TPMPA [(1,2,5,6-tetrahydropyridine-4yl) methyphosphinic acid] were obtained from Sigma; D-AP5 [D(-)-2-amino-5-phosphonopentanoic acid] and GYKI 53655 [1-(4-aminophenyl)-3-methylcarbamyl-4-methyl-7,8-methylenedioxy-3,4-dihydro-5H-2,3-benzodiazepine] were obtained from Tocris Bioscience.

Recording procedures. In wholemount retinas, GC spikes were recorded in a cell-attached mode before breaking the seal. Whole-cell recordings were made from GCs using patch pipettes filled with intracellular solution containing the following (in $\mathrm{mM}$ ): 120 Csgluconate, 10 tetraethylammonium chloride (TEA-Cl), $1.0 \mathrm{CaCl}_{2}, 1.0$ $\mathrm{MgCl}_{2}, 11$ EGTA, and $10 \mathrm{Na}$-HEPES, adjusted to $\mathrm{pH} 7.2$ with $\mathrm{CsOH}$. The calculated reversal potential for $\mathrm{Cl}$ current $\left(\mathrm{E}_{\mathrm{Cl}}\right)$ for this solution was -58 $\mathrm{mV}$. Whole-cell recordings were made from BCs using patch pipettes filled with an intracellular solution containing the following (in mM): 120 Cs gluconate, $1 \mathrm{MgCl}_{2}$, 10 HEPES, 10 TEA-Cl, 10 phosphocreatine$\mathrm{Na}_{-2}, 4 \mathrm{Mg}$-ATP, $0.5 \mathrm{Na}-\mathrm{GTP}$, and 0.1 or $10 \mathrm{EGTA}$, adjusted to $\mathrm{pH} 7.2$ with $\mathrm{CsOH}$. For both BCs and GCs, the intracellular solution was supplemented with $0.05 \%$ Lucifer yellow or sulforhodamine B. Electrodes were pulled from borosilicate glass (1B150F-4; WPI) with a P-97 Flaming/Brown puller (Sutter Instruments) and had a measured resistance of $\sim 7 \mathrm{M} \Omega$. Voltage-clamp recordings were made with either Axopatch 200B or MultiClamp 700B patch-clamp amplifiers (Molecular Devices) using Patchit software (White Perch Software) to generate voltage command outputs and acquire data. Data were filtered at $1 \mathrm{kHz}$ and sampled at $2 \mathrm{KHz}$, digitized, and stored on a PC for off-line analysis. For GC whole-mount recordings, Signal 2 software (CED) was used to generate voltage command outputs, acquire data, control the electrical stimuli, and operate the drug perfusion system. Data were filtered at $5 \mathrm{kHz}$ with a four-pole Bessel filter and were sampled at $15 \mathrm{kHz}$. All procedures were performed at near physiological temperatures $\left(32^{\circ} \mathrm{C}\right)$.

Retinal stimulation. For the experiments involving light stimulation, a series of responses was recorded to a pair of 10-ms-long light flashes with varied ISIs. For GC recordings, the microscope's illuminator was used to deliver a $250 \mu \mathrm{m}$ spot of light that was centered on the GC and focused on the photoreceptor cell layer. An aperture, a series of neutral density filters, and the FN-C LWD condenser (Nikon) were used to control the size, intensity, and focal plane of the stimulus. The tissue was adapted at $30 \mathrm{~cd} / \mathrm{m}^{2}$, and stimulus was $270 \mathrm{~cd} / \mathrm{m}^{2}$. The Michelson contrast was calculated as $C=\left(I_{\max }-I_{\min }\right) /\left(I_{\max }+I_{\min }\right)$, where $I_{\max }$ and $I_{\min }$ are maximum and minimum light intensities, respectively. The stimulation routines were controlled by Signal 2 software (CED). Duration of the light stimulus was controlled by a Uniblitz shutter (Vincent Associates). For BC recordings, full-field light stimuli were evoked using a lightemitting diode (LED; $\lambda_{\text {peak }}=565 \mathrm{~nm}$, HLMP-3950, Agilent) positioned near the microscope stage. Stimulus intensity $\left(1.85 \times 10^{3}\right.$ photons/ $\mu \mathrm{m}^{2} / \mathrm{s}$ ) and duration were controlled by current applied to the LED. Each set of stimuli was separated by a $60 \mathrm{~s}$ interstimulus interval.

For electrical (zap) stimulation, a pair of positive current pulses $(0.1-1$ $\mathrm{ms} ; 3-15 \mu \mathrm{A}$ ) separated by $30 \mathrm{~ms}$ to $60 \mathrm{~s}$ were applied to the $\mathrm{BC}$ dendrites in outer plexiform layer (OPL) (Higgs and Lukasiewicz, 1999) using a patch pipette filled with extracellular solution 1 .

Because a given synapse expresses multiple forms of short-term plasticity, the strength of the stimulus was experimentally adjusted to favor a depression of the putative excitatory transmission. In most cases, the stimulus intensity was increased just above a half-maximal response. Electrically evoked EPSCs (eEPSCs) were recorded from GCs in voltageclamp mode. The stimulating pipette was always inserted into the OPL directly above the GC that was being recorded to preferentially activate a local feedback circuitry.

Identification of morphological types of ganglion and bipolar cells. Each GC was filled with sulforhodamine B, included in a patch pipette solution. In retinal wholemount preparation, the detailed dendritic structure was reconstructed using a Nikon C-1 confocal microscope. A series of 161 images was acquired at $0.5 \mu \mathrm{m}$ steps at $1024 \times 1024$ pixel resolution. A nuclear stain, a mixture of ethidium bromide and ToPro-3 (Invitrogen), was added to aid in determining the thickness of the inner plexiform layer (IPL) and the depth of the GC dendritic stratification (see Fig. $1 A$ ).

In retinal slices, GC fluorescent images were acquired at the end of the recording session, and consecutive optical sections were superimposed to form a single image using MetaMorph (Universal Imaging) and then were processed using Adobe Photoshop 7.0. In both wholemount and slice preparations, the level at which the GC dendritic processes stratified in the IPL was measured as the distance from its processes to the distal margin $(0 \%)$ of the IPL. In general, ON GCs were defined as those whose dendrites stratified $\geq 60-70 \%$ of the IPL depth, and OFF GC stratified $\leq 30-40 \%$ of the IPL depth (Peichl and Wässle, 1981; Sun et al., 2002; Badea and Nathans, 2004; Kong et al., 2005). GCs were distinguished from displaced amacrine cells by their larger soma sizes and the presence of an axon.

BCs were classified as $\mathrm{ON}$ cone BCs, based on their dendritic and axonal morphologies, and the stratification of their somas in the inner nuclear layer and their axon terminals within the ON and OFF sublaminae of the inner plexiform layer (Ghosh et al., 2004). Photomicrographs were recorded using a Photometric Coolsnap ES camera (Roper Scientific) and MetaMorph software (Molecular Devices).

Analyses. Evoked EPSCs and IPSCs were collected, averaged, and analyzed using Tack software (White Perch Software), SigmaPlot (Systat Software), and Clampfit (Molecular Devices). The eEPSC peak amplitude, charge transfer $\left(Q\right.$ in $\left.\mathrm{pA}^{\star} \mathrm{ms}\right)$, and the half-width of the response were computed from the averaged responses. The GC spiking output was collected, averaged, and analyzed using Signal software (CED). The synaptic depression was quantified by calculating a paired-pulse ratio [PPR; second response (R2)/first response (R1)], as illustrated in Figures 2 and 6 . For overlapping responses at short ISIs, the second response was calculated after subtracting the response to a single pulse event. All data are reported as the mean \pm SEM. Statistical significance was determined using either a Student's $t$ test or a paired $t$ test, or, for multiple comparisons, using one-way ANOVA with Scheffé's post hoc analysis. 


\section{Results}

Presynaptic inhibition enhances the reliability of light-evoked ganglion cell output

Presynaptic inhibition from amacrine cells modulates excitatory transmission between ON cone BCs and ON GCs. This presynaptic inhibition-mediated control of neurotransmitter release from the $\mathrm{BC}$ terminal may also reduce synaptic depression. We determined whether lightevoked excitatory inputs to GCs exhibited depression and if so, whether this depression was modified by presynaptic inhibition. In the wholemount retina, we varied the ISI between paired light stimuli to find out (1) whether synaptic depression occurred at the BC to GC synapse, and (2) whether it was shaped by the inhibitory feedback.

We monitored how BC glutamate release affected GC output signals by recording light-evoked extracellular spiking in response to $250-\mu \mathrm{m}$-diameter spots of light that cover the GC's receptive field center (Pang et al., 2003; Völgyi et al., 2005). Pairs of brief light flashes were separated by variable ISIs $(0.03-10 \mathrm{~s})$ with a $60 \mathrm{~s}$ recovery period between each series. We recorded responses in $\mathrm{ON}$-center GCs (Fig. 1A) because $\mathrm{GABA}_{\mathrm{C}} \mathrm{R}$-mediated presynaptic inhibition exerts stronger effects in the ON compared with the OFF visual pathway (Zhang and Slaughter, 1995; Sagdullaev et al., 2006). ON-center GCs were identified based on their response to increments of light (Fig. $1 B$ ) and their characteristic morphology, determined from confocal images (Fig. 1A). To quantify how synaptic depression varied with ISI, we calculated a PPR by dividing the number of spikes during $\mathrm{R} 2$ by the number of spikes during R1. The fractional response was then plotted as a function of ISI (Fig. 1C). We found that responses to the second light stimulus of the pair were reduced to $\sim 65 \%$ of their initial magnitude for the shortest intervals $(65.3 \pm 7.8 \%$, $n=7$ ) (Fig. $1 B, C$ ), consistent with synaptic depression. Response depression was largest when the ISI was short (high frequency) and absent for the longest ISIs (Fig. $1 B, C$ ). The depressed response recovered in $<1 \mathrm{~s}$.

If depression occurs at the BC-to-GC synapse, then the strength of depression may be modulated by BC presynaptic inhibition. To test whether this was the case, we pharmacologically altered the strength of presynaptic inhibition with the $\mathrm{GABA}_{\mathrm{C}} \mathrm{R}$ antagonist TPMPA. In the IPL, TPMPA exclusively acts on BC terminals, but not at GC dendrites (Sagdullaev et al., 2006). In the OPL, GABA receptors do not contribute to light-evoked inhibition (Verweij et al., 1996; Euler and Masland, 2000; McMahon et al., 2004). Upon reducing presynaptic inhibition, the magnitude of depression increased almost twofold (Fig. $1 B, C$ ). The responses to the second light stimulus were now depressed to $\sim 35 \%$ of their initial magnitude for the shortest intervals $(36.0 \pm 8.9 \%, n=7$ ) (Fig. $1 B, C)$. For
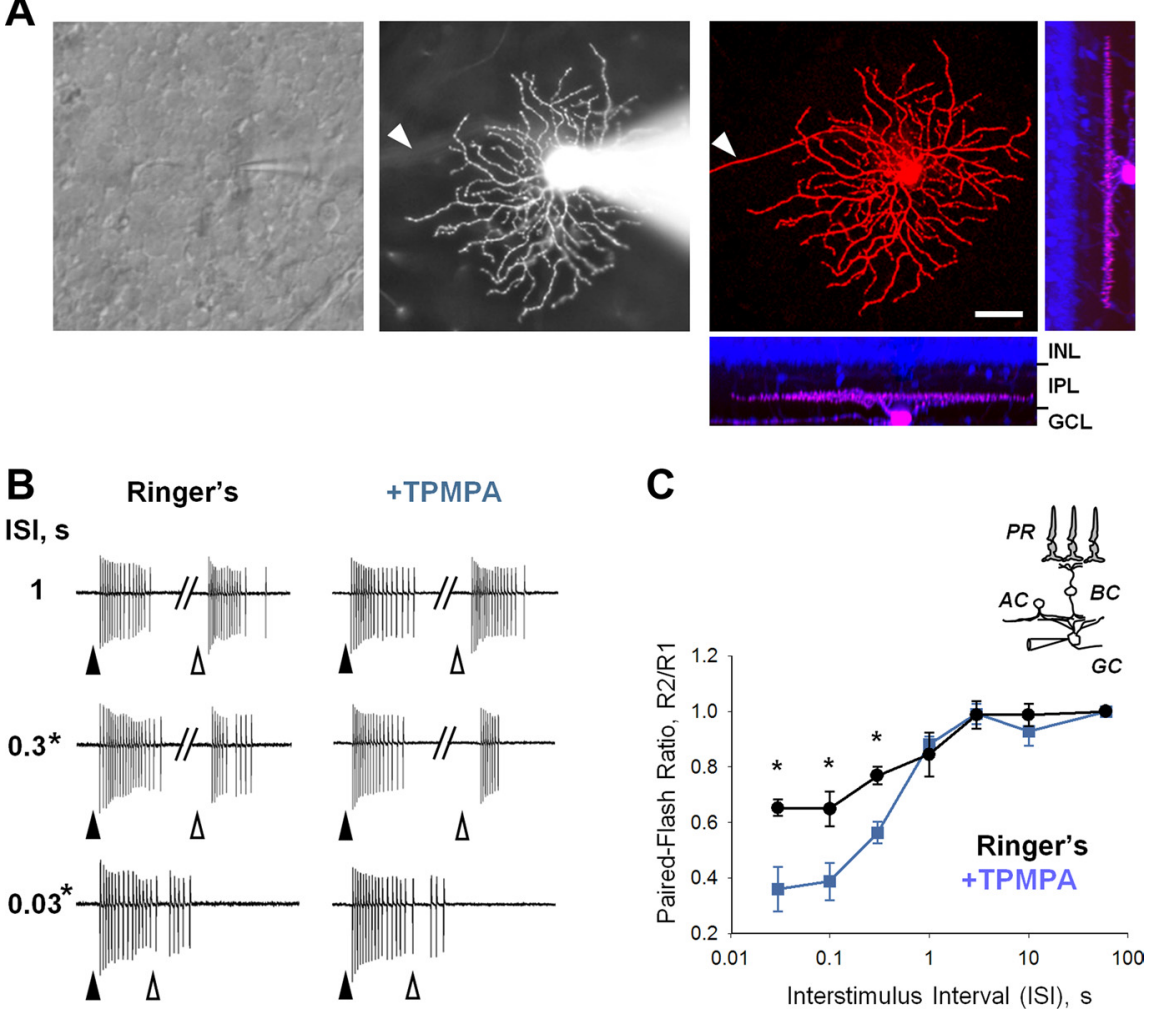

Figure 1. Presynaptic $\mathrm{GABA}_{\mathrm{C}}$ receptors enhance reliability of light-evoked spiking output of $\mathrm{GCS}$ to repetitive stimuli. $\boldsymbol{A}$, The view at the $\mathrm{GC}$ layer in wholemount retinal preparation (left).The outlines of individual cell bodies are visible across the field. The 1024 pixel resolution. A nuclear stain (blue) was added consequently to aid in determining the thickness of the inner plexiform layer and the depth of the dendritic stratification. GCs were distinguished from displaced amacrine cells by the presence of an axon (the $\mathrm{GC}$ shown in $\boldsymbol{A}$. Series of responses were recorded to field. Each series was separated by a 60 s interval. $C$, Paired-flash plasticity of spiking response as a function of ISI in the presence and absence of presynaptic inhibition $(n=7)$. Schematics on the right illustrate the retinal circuitry and stimulation paradigm used sons were made using a paired $t$ test with significance at $p<0.05$. In this and subsequent figures, the data points represent the mean values \pm SEM. The bath temperature for all experiments was maintained at near physiological levels at $32^{\circ} \mathrm{C}$.

ISIs ranging from 0.03 to $0.3 \mathrm{~s}$, depression of the second response was significantly greater after presynaptic inhibition was reduced $(p=0.007, p=0.018, p=0.004$, for $0.03,0.1$ and $0.3 \mathrm{~s}$, respectively, paired $t$ test, $n=7$ ) (Fig. $1 C$ ). These findings are consistent with the notion that presynaptic inhibition limits depression of GC responses.

The increase in synaptic strength in normal Ringer's solution shows that presynaptic inhibition to BCs restricts depression to maintain excitatory transmission to GCs. Our findings strongly suggest that a component of depression occurs at the BC-to-GC synapse. Since these experiments used light stimuli, contributions of photoreceptors or the OPL (Rabl et al., 2006; Singer and Diamond, 2006) could also play a role in the observed GC response decline. Therefore, in the next set of experiments we used electrical stimuli to assess depression at the isolated BC-to-GC synapses.

\section{Presynaptic inhibition reduces paired-pulse depression of} excitatory inputs to GCs

To directly study the plasticity of transmission between BCs and GCs, we bypassed transmission in the outer retina by focally stim- 
ulating an array of BCs with brief electrical pulses in retinal slices (Higgs and Lukasiewicz, 1999; Sagdullaev et al., 2006). This approach mimics the light-evoked input when numerous photoreceptor-driven BCs converge onto a single GC (Higgs and Lukasiewicz, 1999; Chen and Diamond, 2002). BC transmission was assessed by recording eEPSCs from morphologically identified ON GCs, held at $-35 \mathrm{mV}$, to relieve the $\mathrm{Mg}^{2+}$ block of NMDARs (Nowak et al., 1984). We quantified the eEPSCs by measuring their charge transfers $\left(Q_{\text {eEPSC }}\right)$, which largely reflected current flowing through slow-decaying NMDARs ( $\sim 80 \%$ of total Q) (see Fig. 3B) (Cohen, 2000; Sagdullaev et al., 2006).

To determine whether transmission between BCs and GCs showed depression, we electrically activated $\mathrm{BCs}$ with paired constant current stimuli separated by variable ISIs (Fig. $2 A$ ). In the presence of presynaptic inhibition, little depression was observed when the ISI was short $(\leq 0.1 \mathrm{~s})$. Figure $2 D$ shows that with inhibition present the second eEPSCs were only modestly depressed when the ISI was short, consistent with only minimal depression (Fig. $2 A$ ). When the ISI was increased (ISIs $0.3-3 \mathrm{~s}$ ), a more sizeable amount of depression was revealed. These findings show that synaptic depression was minimal when inhibition was intact, which is in agreement with our observations with paired light stimuli (Fig. 1).

To determine whether $\mathrm{GABA}_{\mathrm{C}} \mathrm{R}$-mediated presynaptic inhibition reduced synaptic depression, we compared paired-pulse depression before and after the blockade of presynaptic inhibition with TPMPA. Figure 2, $A$ and $B$, shows that the second eEPSC response was now significantly more depressed when glutamate release was no longer limited by presynaptic inhibition. Thus, presynaptic inhibition limited depression of the eEPSCs, just as we observed for depression of paired light-evoked responses. The similarities in our findings for electrically and lightevoked responses suggest that most of the depression occurs at the BC-to-GC synapse, and not in the OPL.

\section{Distinct synaptic depression patterns are attributed to different effects of presynaptic inhibition on NMDAR and AMPAR signaling}

The eEPSCs in ON GCs consist of rapid AMPAR-mediated and slower NMDAR-mediated components (Figs. $2 B, C$ ). To isolate the AMPAR responses, we blocked the activation of NMDARs with D-AP5 (Fig. 2C). We compared how presynaptic inhibition affected depression of the AMPAR and NMDAR eEPSC components. Figure 2, $D$ and $E$, shows that when presynaptic inhibition was blocked by TPMPA and when the ISI was short, both NMDAR- and AMPAR-mediated eEPSCs were more depressed, suggesting that presynaptic inhibition limits depression. However, the effects that presynaptic inhibition exerted upon the AMPAR- and NMDAR-mediated components were strikingly different. For short ISIs, the second NMDAR-mediated eEPSCs (Fig. $2 B, D)(p=0.001,0.0001$, and 0.036 for ISIs of $0.03,0.1$, and $0.3 \mathrm{~s}$, respectively, paired $t$ test, $n=9$ ) were significantly less depressed compared with AMPAR-mediated eEPSCs (Fig. 2C,E) $(p=0.04,0.04$, and 0.08 for ISIs of $0.03,0.1$, and $0.3 \mathrm{~s}$, respectively, paired $t$ test, $n=6$ ). AMPAR-mediated responses recovered from depression with a time constant of $0.32 \pm 0.10 \mathrm{~s}$ (estimated from exponential fit to curve in Fig. $2 E ; n=6$ ). In contrast, the shape of the paired-pulse response curve for NMDAR activation was more complex and was approximated by two phases (Fig. 2D). The late phase, at ISIs $>1 \mathrm{~s}$, was very similar to an AMPAR profile with a time constant of recovery of $\sim 0.3 \mathrm{~s}$. However, the early phase, at ISIs $<1 \mathrm{~s}$, little, if any depression was

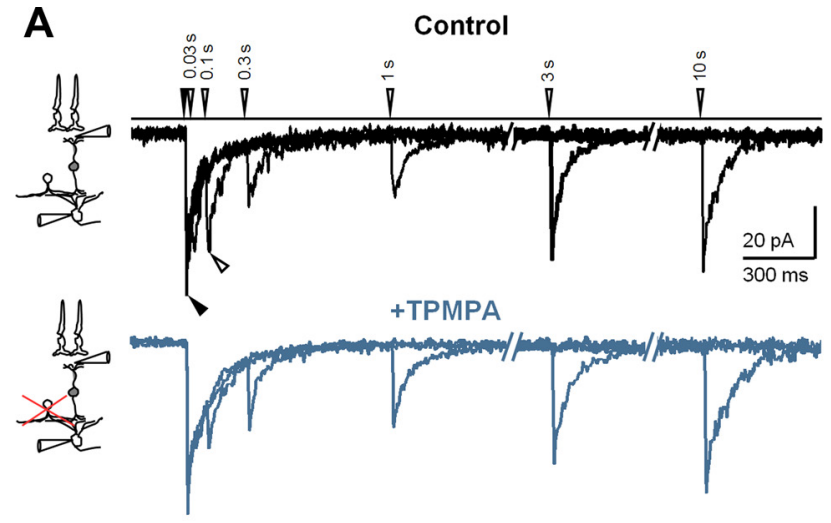

B
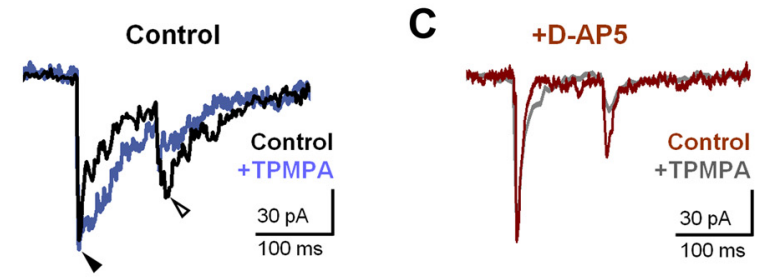

D
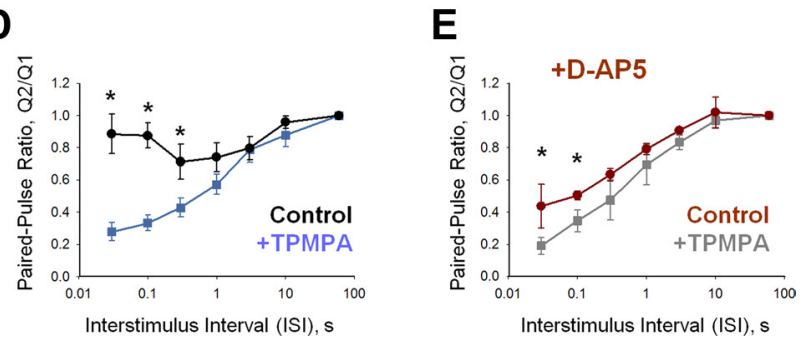

Figure 2. Activation of presynaptic $G_{B B} A_{C}$ receptors selectively suppresses the initial but not secondary response at short ISIs. $A$, The excitatory output of retinal $B C s$ to two electrical stimuli separated by variable ISIs was recorded from morphologically identified ON GC. The holding potential $\left(V_{h}\right)$ was $-35 \mathrm{mV}$ to examine both NMDAR and AMPAR components. The average $P P R, Q 2 / Q 1$, was measured at each ISI. Schematics on the left illustrate the retinal circuitry and stimulation paradigm used to elicit and record electrically evoked responses in GCs in a retinal slice. Inputs to BCs were directly activated by a brief electrical pulse (top electrode). Evoked EPSCs were recorded from the corresponding ON GC (bottom electrode). Representative eEPSCs evoked by pairs of stimuli with variable ISIs recorded in control conditions and in the absence of $\mathrm{GABA}_{c}$-mediated presynaptic inhibition (+TPMPA, $50 \mu \mathrm{m}$ ). The arrowheads denote the time of the first (filled) and the second (open) electrical pulses. The stimulus artifacts at response onset were removed for clarity. When presynaptic inhibition is present, depression in the second response of the pair is reduced. $\boldsymbol{B}, \boldsymbol{C}$, Superimposed responses in the presence and absence of presynaptic inhibition for total (mostly NMDAR) $(\boldsymbol{B})$ and isolated AMPAR-mediated $(\boldsymbol{C})$ current at a $100 \mathrm{~ms} I S I$. D, E, Paired-pulse plasticity profiles (Q2/Q1, as a function of ISI) in the presence and absence of presynaptic inhibition for total $(n=10)(\boldsymbol{D})$ and AMPAR $(n=5)(\boldsymbol{E})$ eEPSCs. Evoked EPSCs were isolated using a cocktail of inhibitory blockers ( $100 \mu \mathrm{m}$ bicuculline, $5 \mu \mathrm{m}$ strychnine). Statistical comparisons were made using paired $t$ tests with significance at $p<0.05$.

observed with NMDARs compared with the marked depression exhibited by AMPARs.

The distinct depression profiles could be explained by presynaptic inhibition differently shaping AMPAR- and NMDARmediated eEPSCs. We compared how presynaptic inhibition affected AMPAR- and NMDAR-mediated eEPSCs in the presence of selective antagonists GYKI-53655 (40 $\mu \mathrm{M})$ or D-AP5 (50 $\mu \mathrm{M})$, respectively. To quantify a fast desensitizing AMPARmediated response, we measured the peak amplitude. Since the NMDAR-mediated component decays much more slowly and accounts for most of the EPSC current, we estimated its contribution from the charge transfer (Chen and Diamond, 2002; Sagdullaev et al., 2006) (Fig. 3A). Blockade of $\mathrm{GABA}_{\mathrm{C}} \mathrm{R}$ mediated 


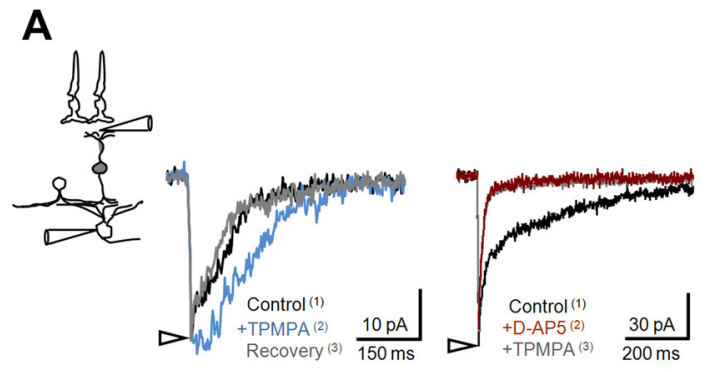

C

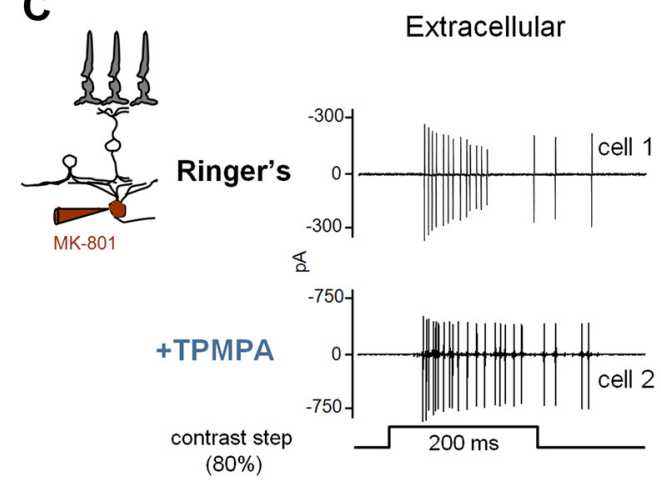

D

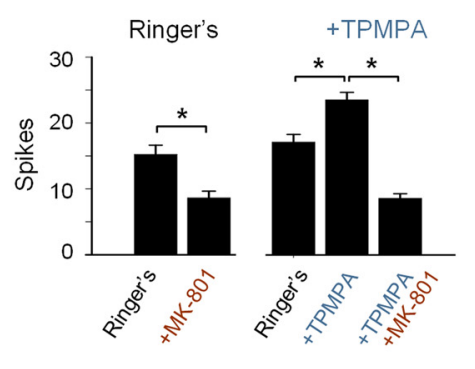

Intracellular $(+$ MK-801)

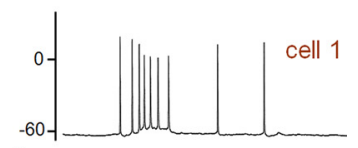

ऐे

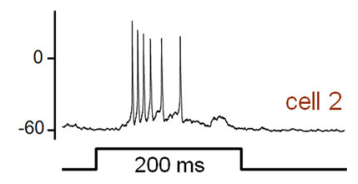

$\mathbf{E}$

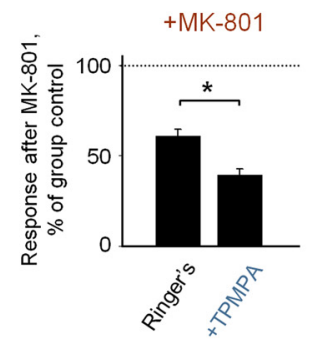

Figure 3. Presynaptic inhibition has differential effects on AMPA and NMDA receptor-mediated GC input/output profiles. $A, G A B A_{C} R$-mediated inhibition reduces glutamate release from the $B C$ terminal and controls excitatory input to GCs. eEPSCs were recorded from ON GCs held at $-35 \mathrm{mV}$. Blockade of GABA ${ }_{C} R s$ with TPMPA (50 $\left.\mu \mathrm{m}\right)$ significantly increased eEPSCs, which recovered back to control levels. Presynaptic inhibition had a selective effect on activation of NMDAR-mediated eEPS(s as its effect was abolished when only AMPARs mediated the response (+ D-AP5, $50 \mu \mathrm{m}$ ). $\boldsymbol{B}$, Summary graph shows the effects of TPMPA on individual excitatory inputs to GC. Responses of individual GCs following application of TPMPA were averaged for each condition (as indicated on $x$-axis) and then normalized to their pre-TPMPA values (dashed line). C, Light-evoked spiking responses from ON GCs in normal Ringer's solution $(n=8)$ and in the presence of TPMPA (100 $\mu$, $n=$ 5). As shown in the illustration, the pipette solution contained MK-801 (1 mM). In each group, spiking responses were first obtained in a loose-patch configuration (extracellular, group controls). For all cells, the contribution of NMDAR-mediated input to GC spiking output was consequently evaluated by infusing MK-801 in a whole-cell configuration (intracellular). $\boldsymbol{D}$, Application of MK-801 within the GC significantly suppressed light-evoked spiking in both groups $[p=0.002(n=8)$ and $p=0.004(n=5)$, repeated-measures ANOVA], with significantly larger effect on GCs pretreated with TPMPA $(p=0.004, E)$.

inhibition with TPMPA-enhanced eEPSC charge transfer (Fig. $3 A, B)\left(Q_{\text {eEPSC }}\right.$ ratio $\left.=1.78 \pm 0.20, n=10, p \ll 0.01\right)$, which largely reflects NMDAR activation (Sagdullaev et al., 2006). The peak eEPSC amplitude, which largely reflects AMPAR activation, was not affected by TPMPA, consistent with the slow activation kinetics of $\mathrm{GABA}_{\mathrm{C}}$ Rs (Fig. $3 A, B$ ) (amplitude of eEPSC ratio = $1.05 \pm 0.12, n=10, p>0.05$ ) (Eggers and Lukasiewicz, 2006b). The blockade of $\mathrm{GABA}_{\mathrm{C}} \mathrm{Rs}$ did not significantly affect isolated AMPAR-mediated currents (Fig. $3 A, B)\left(D-A P 5, Q_{\text {eEPSC }}\right.$ ratio $=$ $0.91 \pm 0.10, n=10, p>0.05)$, but markedly enhanced NMDARmediated currents (Fig. 3B) (GYKI-53655, $Q_{\text {eEPSC }}$ ratio $=1.71 \pm$ $0.14, n=10, p \ll 0.01)$.

Although these experiments show that presynaptic inhibition selectively affected the NMDAR-mediated inputs to GCs, it is also important to know how $\mathrm{GABA}_{\mathrm{C}} \mathrm{R}$-mediated modulation of NMDAR activity contributes to light-evoked GC spiking output in the intact network. To address this, we recorded light-evoked spiking responses of ON GCs in the wholemount retina either when presynaptic inhibition remained intact or when presynaptic inhibition was blocked with TPMPA. To avoid comparisons between different classes of GCs, we selected GCs with similar initial response properties $(15.24 \pm 1.39$ and $16.33 \pm 1.62$ spikes, $p=0.4$ ). Blocking presynaptic inhibition augmented the GC spiking responses from $16.33 \pm 1.62$ to $23.01 \pm 1.84$ spikes (Fig. $3 D)(p=0.003, n=5$, repeated-measures ANOVA), consistent with our eEPSC data (Fig. $3 A, B$ ).

To determine how presynaptic inhibition affects the NMDAR component of the light responses, we assessed responses before and after blockade of NMDARs with presynaptic inhibition either intact or blocked. To avoid potential network effects of NMDAR inhibitors, we selectively blocked NMDARs in the recorded GC by including MK-801 (1 mM) in the recording pipette. To assess the NMDAR contribution to the light responses, we first recorded extracellular spiking in loose-patch mode. Then, after rupturing the patch membrane, intracellular MK-801 blocked NMDARs. After $10 \mathrm{~min}$, spiking responses were recorded in whole-cell current-clamp mode and compared with extracellular responses (Fig. 3C). MK-801 significantly reduces light-evoked GC spiking in both groups (Fig. 3D), consistent with the large role of NMDARs in spiking responses. Furthermore, the effect of MK-801 was largest when presynaptic inhibition was removed (Fig. 3E). With presynaptic inhibition intact, MK-801 reduced spiking to $60.76 \pm 3.8 \%$ of control value (from $15.24 \pm$ 1.39 to $8.64 \pm 1.39, p=0.002, n=8$, paired $t$ test). When presynaptic inhibition was blocked, MK-801 reduced spiking to $39.20 \pm 3.4 \%$ of control value (from $23.01 \pm 1.84$ to $8.85 \pm 0.66$, $p=0.004, n=5$, repeated-measures ANOVA). Therefore, NMDARs were activated to a larger extent in the absence of presynaptic inhibition. These findings support the hypothesis that $\mathrm{GABA}_{\mathrm{C}} \mathrm{R}$-mediated inhibition limits activation of NMDARs, consistent with our earlier report (Sagdullaev et al., 2006). Furthermore, there were no differences between the +MK-801 group and the +MK-801+TPMPA group, indicating that the isolated AMPAR component was not enhanced by TPMPA. Similar findings obtained with either bath-applied D-AP5 or intracel- 

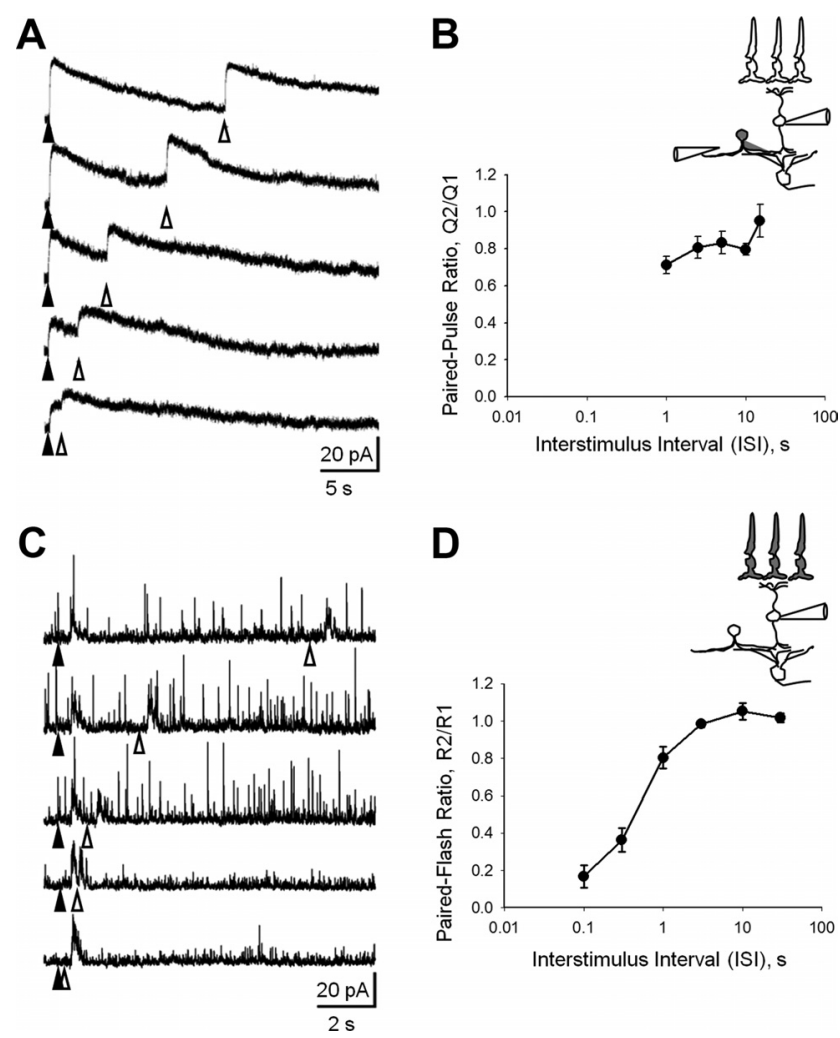

Figure 4. Inhibition to 0 N cone bipolar cells shows paired-pulse depression. $\boldsymbol{A}$, Representative zap-evoked GABAc currents from $0 \mathrm{~N}$ cone $\mathrm{BC}$ in response to pairs of zaps (gray dots) delivered to the IPL adjacent to $B C$ axon terminals. Zap stimuli were $2 \mathrm{~ms}$ in duration and had amplitudes ranging from 3 to $7.5 \mu \mathrm{A}$, consistent within a cell. Zap artifacts were removed for clarity. $\boldsymbol{B}$, When the interval between zaps was decreased, $\mathrm{GABA}_{C}$ current showed depression ( $n=5$ cells). C, Representative L-IPSCs from ON cone BCs, in response to pairs of brief (10 ms) light stimuli. When the interval between light stimuli was $\geq 3 \mathrm{~s}$, the L-IPSC s showed no depression. However, when the interval between light stimuli was decreased, the L-IPSCs showed robust depression. $D$, Summary data from five cells. Schematics on the right illustrate the retinal circuitry and stimulation paradigms used to elicit and record electrically evoked $(\boldsymbol{A})$ and lightevoked $(\boldsymbol{C})$ responses in $\mathrm{BC}$.

lular MK-801 demonstrate that the network effects of NMDAR blockade were minimal.

\section{Inhibitory input to bipolar cells shows synaptic depression}

When presynaptic inhibition is present, synaptic depression was reduced and the second excitatory response at short ISIs was strong (Figs. 1,2). Because presynaptic $\mathrm{GABA}_{\mathrm{C}}$ Rs limit excitatory transmission to GCs, enhanced excitation may also be attributable to reduced presynaptic inhibition. To find out whether the enhanced second response observed with short ISIs was attributable to reduced presynaptic inhibition, we determined whether BC IPSCs exhibited synaptic depression. We directly assessed the inhibitory synapse performance by evoking GABA release from amacrine cells using an extracellular stimulating electrode placed in the IPL. GABA release was assayed by recording $G_{A B A} R$ mediated IPSCs from ON cone BCs. The GABA $\mathrm{C}_{\mathrm{R}}$ component was pharmacologically isolated by including bicuculline and strychnine in the bath to block $\mathrm{GABA}_{\mathrm{A}} \mathrm{R}$ and glycine receptor (glycineR) response components, respectively. We varied the ISI between paired electrical stimuli to measure the frequency dependency of eIPSCs. Figure $4 A$ shows representative electrically evoked responses when using this paradigm. The response to the second stimulus was diminished as the ISI was decreased (compare upper and lower traces), consistent with paired-pulse de- pression of transmission between amacrine cells and BCs (Fig. $4 A, B)$. Because of the slow onset and offset of the $\mathrm{GABA}_{\mathrm{C}} \mathrm{R}$ eIPSCs, we were unable to reliably determine the second response for shorter ISIs. Nevertheless, these data support the notion that the enhancement of the second excitatory response observed in GCs (Fig. 2 B) was attributable to reduced presynaptic inhibition.

Comparable results were obtained with light-evoked IPSCs (L-IPSCs) recorded from ON cone BCs. Because physiologically relevant stimuli were used and $\mathrm{GABA}_{\mathrm{A}}$ Rs and glycineRs were not blocked, the dominant $\mathrm{GABA}_{\mathrm{C}} \mathrm{R}$ component was briefer than the electrically evoked current, enabling second response measurements for shorter ISIs. The smaller $\mathrm{GABA}_{\mathrm{C}} \mathrm{R}$ component is attributable to serial inhibition within the amacrine cell network (Eggers and Lukasiewicz, 2006a). When serial inhibition is blocked by the inclusion of bicuculline and strychnine in the bathing solution, the magnitude of inhibition is enhanced (Fig. 4A) (Eggers and Lukasiewicz, 2006a). Figure 4, $C$ and $D$, shows that paired-pulse depression was greatest for short ISIs, supporting the argument that reduced presynaptic inhibition contributed to the prolonged second EPSCs. The reduced initial responses and the reduced spontaneous activity in lower traces in Figure $4, A$ and $C$, were likely attributed to rundown during prolonged sessions because these responses were recorded last. The similar results obtained with and without the use of $G_{A B A} R$ and glycineR blockers (Fig. $4 A, C$ ) suggest that serial interactions do not significantly contribute to paired-pulse depression of the $\mathrm{GABA}_{\mathrm{C}} \mathrm{R}$-mediated IPSCs.

\section{Synaptic depression occurs presynaptically}

Since synaptic depression affected both AMPAR- and NMDARmediated response components, these findings suggest a common presynaptic mechanism, as described for the rod bipolar-AII amacrine cell synapse (Singer and Diamond, 2006; Dunn and Rieke, 2008). However, our data do not rule out an additional postsynaptic contribution to depression. Previous work has demonstrated that both presynaptic (von Gersdorff and Matthews, 1997) and postsynaptic (Trussell et al., 1988, 1993; Brenowitz and Trussell, 2001) mechanisms can cause synaptic depression. In synapses with high release probability (Pr), including photoreceptors and rod BC ribbon synapses, a presynaptic mechanism, and not a postsynaptic receptor mechanism, is a major cause of depression (Rabl et al., 2006; Singer and Diamond, 2006). Thus, we wanted to evaluate the relative contributions of presynaptic and postsynaptic mechanisms to depression in the synapse between BCs and GCs.

Presynaptic depression is frequently attributed to vesicle depletion. If vesicle depletion is the major determinant of depression at the $\mathrm{BC}-\mathrm{GC}$ synapse, then the plasticity profile of this synapse should be well described by the transmitter depletion model (O’Donovan and Rinzel, 1997; Tsodyks and Markram, 1997). In this model, if the depletion of vesicles is the sole contributor to depression, then eEPSCs will reach common, steadystate amplitudes during high-frequency stimulus trains that are independent of their initial response amplitude. The steady-state response occurs when the rate of vesicle depletion equals the rate of vesicle replenishment. In contrast, if postsynaptic receptor desensitization is the major determinant of synaptic depression, then the responses to a stimulus train will progressively decline as Pr is increased. To test whether the depression of the GC eEPSCs was consistent with the vesicle depletion model, we evaluated their responses to trains of 10 pulses delivered to BCs, each separated by $300 \mathrm{~ms}$. This stimulation interval (Fig. 5A1) was significantly shorter than the measured recovery time constants for 
A1

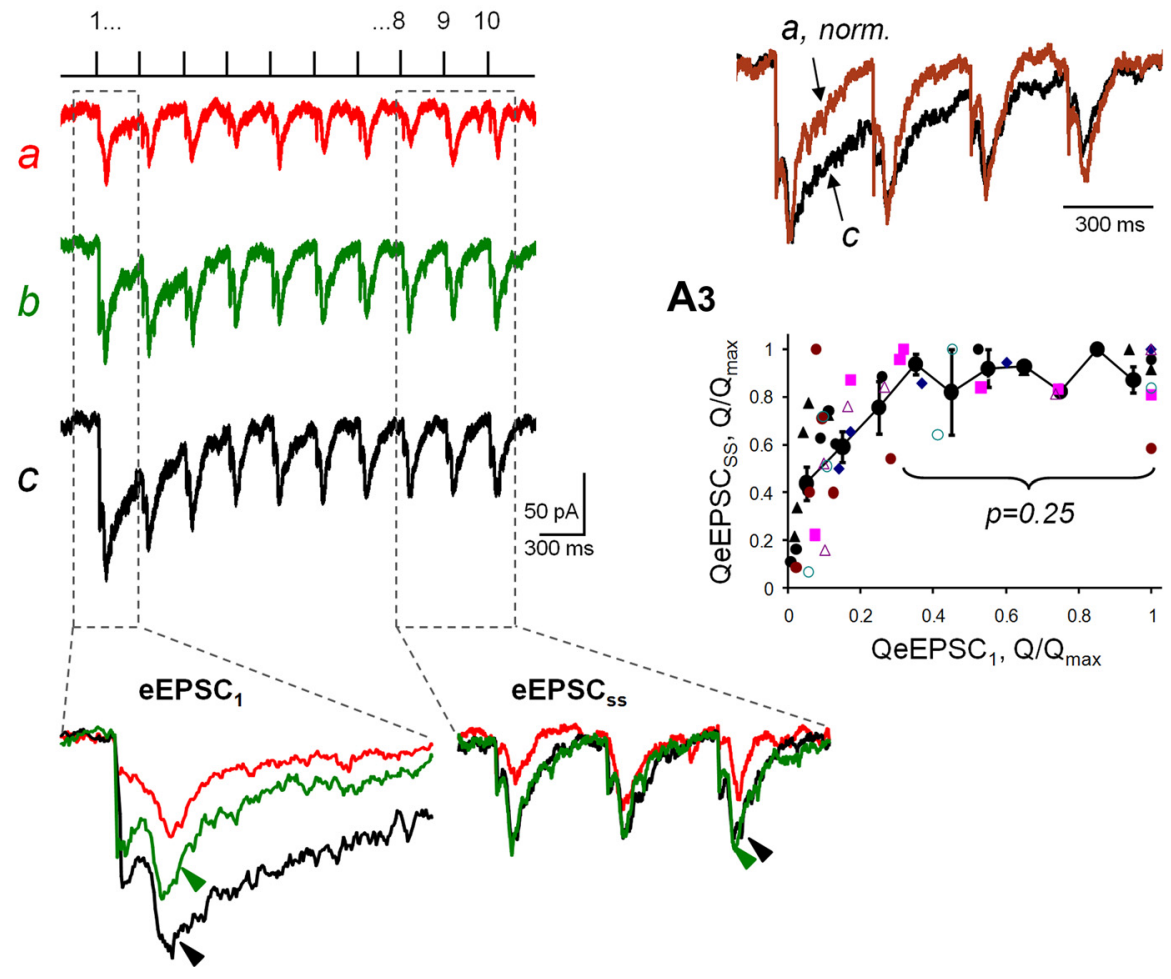

B
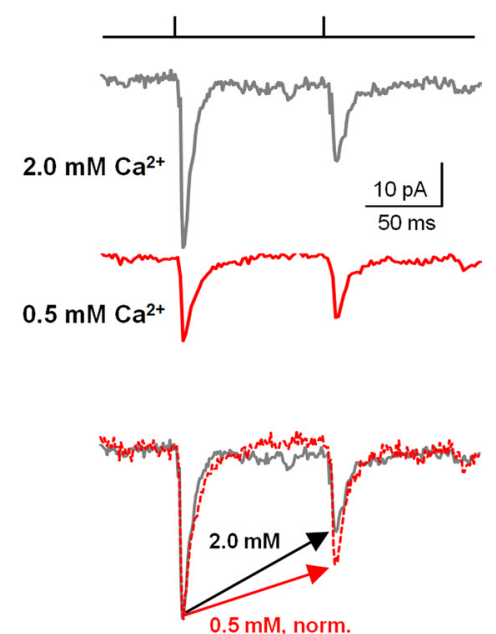

Figure 5. Lowering Pr reduces depression. A, eEPSCs evoked by trains of 10 consecutive electrical stimuli with $300 \mathrm{~ms} I S \mathrm{I}$ at different stimulus intensities. Representative traces illustrate responses at low (a), medium (b), and high (c) intensities (of seven used). The traces above the responses in $\boldsymbol{A} \mathbf{1}$ and $\boldsymbol{B} \mathbf{1}$ illustrate the stimulus paradigms. Recording conditions are as in Figure $2 A$ (bicuculline, strychnine, TPMPA, $\left.V_{h}-35 \mathrm{mV}\right)$. STD was not evident when Pr was low (A1, trace a), but was prominent when Pr was high (A1, trace c). Raising the strength of the stimulus resulted in significant enhancement of the initial response, eEPSC1 (inset, left), while reaching a plateau for steady-state responses (eEPSC $\mathrm{SS}_{\mathrm{S}}$, as highlighted by green and black arrowheads (inset, right). $\mathbf{A 2}$, The first four eEPSCs, traces a and c, from $\boldsymbol{A} \boldsymbol{1}$ (normalized to eEPSC1 in trace c) illustrate the decrease in STD when Pr was low. A3, Response $Q_{\text {eepscss }}$ values (averages of eEPSCs $8-10$, normalized to their corresponding peak value, $Q_{\text {eEPscss }}$ max) plotted as a function of initial response (eEPSC1, normalized to their corresponding peak value, $Q_{\text {eEPsC } 1}$ max). Colored symbols represent trains from seven individual GCS. Filled black circles connected by a solid line represent the average values for all seven GCS. B, The response from a representative GC $(n=4)$ of the isolated AMPAR-mediated eEPSC $(\mathrm{Bic}+\mathrm{STR}+\mathrm{TPMPA}+\mathrm{D}-\mathrm{AP5})$ to a pair of electrical stimuli separated by $100 \mathrm{~ms}$ is shown in normal $(2.0 \mathrm{~mm})$ (top) and in low $(0.5 \mathrm{~mm})$ extracellular $\mathrm{Ca}^{2+}$ (middle). For comparison, responses normalized to a conditioning pulse are superimposed for comparison in low and normal $\mathrm{Ca}^{2+}$ (bottom).

both AMPAR- and NMDAR-mediated currents (1.03 and $2.61 \mathrm{~s}$, respectively). Pr was altered by varying the strength of the electrical stimulus (Fig. 5A1, traces a to c, weak to strong). As expected, there was less depression when the first eEPSC ( eEPSC $_{1}$ ) was small and Pr was low (Fig. 5A1, trace a). Furthermore, there was more depression when eEPSC 1 was large and $\mathrm{Pr}$ was high (Fig. 5A1, trace c). This shift to depression becomes obvious when we normalize the responses to weak stimuli in trace a to the responses to strong stimuli in trace $c$, as shown in Figure 5A2. Both presynaptic and postsynaptic mechanisms could explain these findings because reducing Pr prevents depletion of the readily releasable pool (RRP) of vesicles, and also results in less postsynaptic receptor desensitization and/or saturation, resulting in more robust responses.

To distinguish between the presynaptic and postsynaptic depression, we determined how well our data were described by the transmitter depletion model. In Figure $5 A 1$ (insets), the responses of the GC to a train of pulses are superimposed for comparison. Across a wide range of stimuli, increasing stimulus strength enhanced the initial response amplitude, defined as EPSC $_{1}$ (Fig. 5A1, left). The amplitude of the EPSC steady-state response $\left(\mathrm{EPSC}_{\mathrm{SS}}\right)$, determined from the final three pulses within the train, depended on the initial response amplitude (Fig. 5A1, compare red and black races in left and right panels). The magnitude of this steady-state response is the amount of the replenished neurotransmitter during the given $0.3 \mathrm{~s}$ interval. If postsynaptic receptor desensitization was involved, then it would prevent the function from reaching a plateau by contributing to a decline in the $\mathrm{EPSC}_{\mathrm{SS}}$.

To compare these data across all cells $(n=7)$, we plotted the magnitude of the $Q_{\text {eEPSCSS }}$ as a function of initial response $\left(Q_{\mathrm{eEPSC1}}\right)$ (Fig. 5A3). As for GCs in Figure 5A1, we found that when $Q_{\mathrm{eEPSC} 1}$ is small ( $<35-40 \%$ of its maximum value), reflecting a low $\mathrm{Pr}$, then the eEPSC $_{\mathrm{SS}}$ value increases, consistent with an increased recruitment of vesicles into the RRP (Fig. 5A3). However, for larger values of $Q_{\text {eEPSC1 }}(40-100 \%$ of maximum), reflecting a higher $\mathrm{Pr}$, the size of $\mathrm{eEPSC}_{\mathrm{SS}}$ reaches a stable, plateau level, despite further increases in initial Pr and eEPSC1. Since a plateau in $\mathrm{eEPSC}_{\mathrm{SS}}$ reflects the balance between the rate of the vesicle depletion and replenishment, our findings are consistent with a purely presynaptic depletion model of synaptic depression (Brenowitz et al., 1998). If postsynaptic receptor desensitization also contributed to synaptic depression, then eEPSC $_{\mathrm{SS}}$ would decrease with increasing $\mathrm{Pr}$, as demonstrated for the nucleus magnocellularis synapse (Brenowitz and Trussell, 2001). The plateau in eEPSC $_{\mathrm{SS}}$ with increasing Pr strongly suggests that postsynaptic receptor desensitization did not play a major role in depression for bipolar to GC transmission.

Although our findings support a presynaptic model of depression, increases in electrical stimulation may have not only increased $\mathrm{Pr}$ in individual BCs, but also may have recruited additional BC inputs to the GC. To rule out the latter possibility, 
A

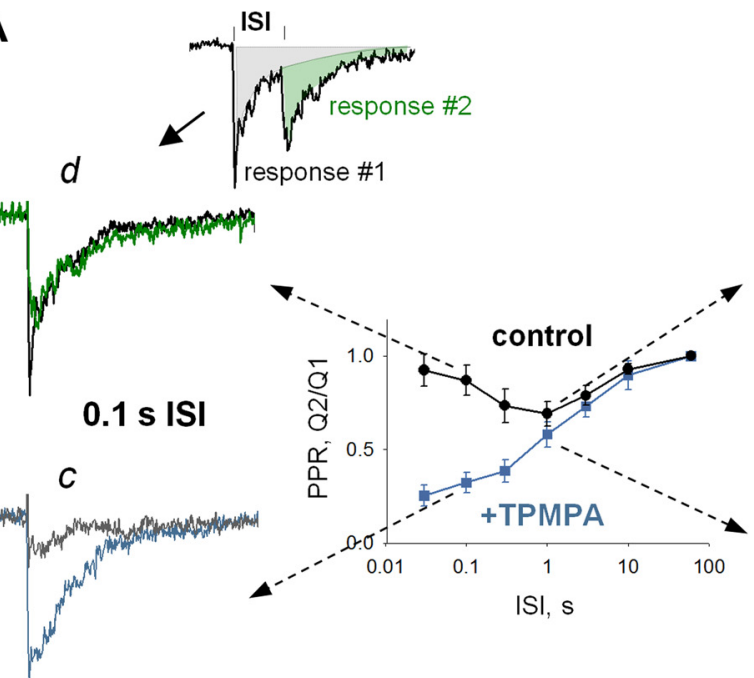

B

\section{$0.1 \mathrm{~s} I S I$}

control $\frac{1}{100 \mathrm{~ms}}$
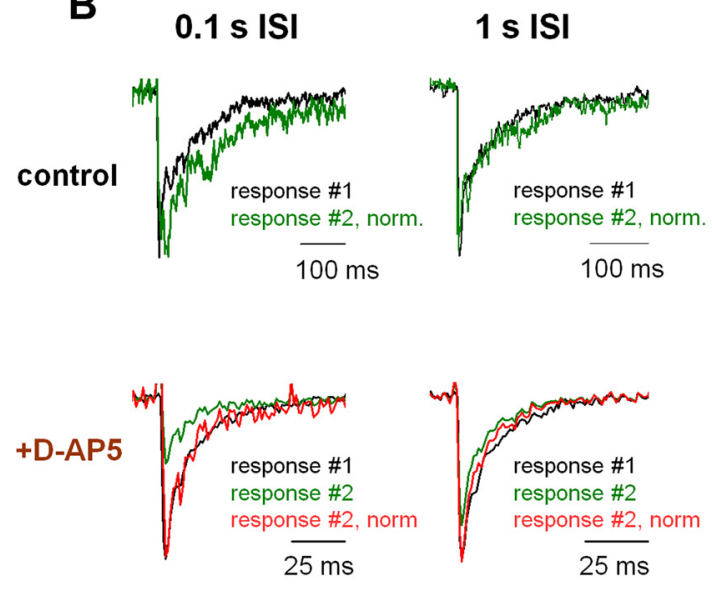

C
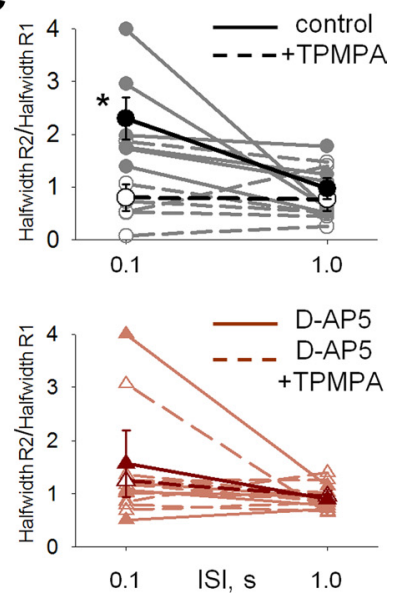

Figure 6. Interaction between excitation and presynaptic inhibition is dynamic and depends on time between two evoked events. $A$, The eEPSCs from GCs to paired-pulse stimulation in the presence (control, inhibition) and absence of GABA $R$ R-mediated presynaptic inhibition (+TPMPA, no inhibition) were superimposed for short $(0.1 \mathrm{~s} ; \boldsymbol{c}$ and $\boldsymbol{d})$ and long $(1 \mathrm{~s} ; \boldsymbol{a}$ and $\boldsymbol{b})$ ISIs for comparison. When paired responses overlapped for short ISIs (cand $\boldsymbol{d}$ ), the response to a second pulse was obtained by subtraction of the average initial eEPSC. $B, G A B A_{C} R$-mediated presynaptic inhibition fails to limit glutamate release from the $B C$ terminal to a second pulse for short ISIs ( 0.1 s, control left), prolonging the eEPSC (decay) time course because of enhanced spillover activation of perisynaptic NMDARs. In contrast, inhibition effectively limited EPSCs evoked by both pulses when the ISI was longer (1 s, control, right). GABA $\mathrm{R}$-mediated inhibition did not affect the time course of AMPAR-mediated eEPSCS (+ D-AP5). C, Total (upper) and AMPAR-mediated (lower) eEPSC half-widths plotted as a function of ISI, in the presence or absence of presynaptic inhibition. Individual cell half-widths are shown in pale colors, and average half-widths are shown in solid colors.

we activated BC inputs with fixed electrical stimuli and then varied Pr by changing the concentration of extracellular $\mathrm{Ca}^{2+}$. To study the direct effects of varying $\mathrm{Pr}$, we pharmacologically isolated the AMPAR component of the eEPSC. This avoided effects of altered extracellular $\mathrm{Ca}^{2+}$ on NMDARs and inhibitory inputs onto GCs. In control conditions $\left(2.0 \mathrm{mM} \mathrm{Ca}^{2+}, 1.0 \mathrm{mM} \mathrm{Mg}^{2+}\right)$, eEPSCs in GCs showed strong depression when we stimulated BC inputs with a pair of pulses separated by $100-300 \mathrm{~ms}$ (Fig. 5B). By contrast, depression was greatly reduced, when extracellular $\mathrm{Ca}^{2+}$ was lowered $\left(0.5 \mathrm{~mm} \mathrm{Ca}^{2+}, 2.5 \mathrm{~mm} \mathrm{Mg}^{2+}\right)$ (Fig. $5 B$, bottom, normalized to conditional stimulus and superimposed responses). Further, because the time course of eEPSCs did not change when calcium concentrations were altered (compare scaled initial responses), postsynaptic AMPAR desensitization is unlikely to contribute to the depression observed here. Together, these data suggest that presynaptic depletion of vesicles is a significant source of depression

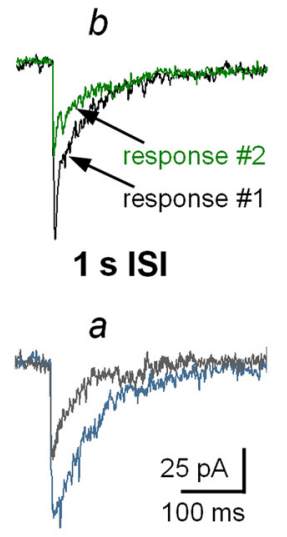

and that lowering the initial Pr and minimizing vesicle depletion reduced synaptic depression.

\section{Nonlinear modulation of NMDAR activation by presynaptic inhibition} During paired-pulse stimulation, the extent of spillover activation of perisynaptic NMDARs depends upon the strength of presynaptic inhibition during both conditioning and test stimuli. We showed in Figure 4 that $\mathrm{GABA}_{\mathrm{C}} \mathrm{R}$-mediated inhibition was selectively reduced during the second response compared with the first response. This explains the nonlinear NMDAR plasticity profile at short ISIs (Fig. 2D), since this would increase glutamate release and spillover activation of perisynaptic NMDARs in the second response, leading to less depression at short ISIs (Fig. $2 B, D$ ). In contrast, activation of synaptic AMPARs is not directly limited by $\mathrm{GABA}_{\mathrm{C}} \mathrm{R}$-mediated presynaptic inhibition (Fig. $3 A$ ), leading to a relatively small reduction in depression in the presence of presynaptic inhibition (Fig. 2E).

We hypothesized that the differential effects of $\mathrm{GABA}_{\mathrm{C}} \mathrm{R}$-mediated presynaptic inhibition on the paired-pulse depression profiles of NMDAR and AMPAR activation (Fig. $2 D, E$ ) can be explained by diminished spillover activation for NMDARs, but not for AMPARs. If this is true, then the decay of the excitatory NMDAR component during the second pulse should increase, attributable to enhanced spillover, reflecting reduced presynaptic inhibition, similar to the effect of TPMPA (Fig. 3A). To test this prediction, we compared the decay of the eEPSCs evoked by the first stimulus to the time course of eEPSCs elicited by the second stimulus (Fig. 6A, inset). Reducing presynaptic inhibition should slow the eEPSC decay, attributable to enhanced activation of NMDARs. We compared the total (predominantly NMDARmediated) eEPSCs at two ISIs, from the late (ISI = $1 \mathrm{~s}$ ) and early $($ ISI $=0.1 \mathrm{~s})$ phases of the plasticity curves. For each ISI, eEPSCs recorded in the absence or in the presence of presynaptic inhibition were superimposed (Fig. 6Aa-d).

The effects of presynaptic inhibition upon depression depended on the ISI. At an ISI of $1 \mathrm{~s}$, in the absence of presynaptic inhibition the initial Pr was high, significantly reducing the second response (Fig. 6 Aa). In the presence of presynaptic inhibition that lowered the initial Pr (Fig. 6Ab), the second total response was still reduced (Fig. $6 \mathrm{Ab}$ ), suggesting that for a $1 \mathrm{~s}$ ISI the presynaptic inhibition had little effect on synaptic depression. At $0.1 \mathrm{~s}$ ISIs, in the absence of inhibition, there is even stronger depression of the paired responses (Fig. $6 \mathrm{Ac}$ ). In contrast to that observed for $1 \mathrm{~s}$ ISIs, depression of the second response was dramatically reduced when the initial release was diminished by presynaptic inhibition (Fig. 6Ad). In fact, with short ISIs and intact 
presynaptic inhibition, the time course of the second response was significantly prolonged, compared with the initial response (Fig. $6 B, C$, top panels) (halfwidth ratio $\mathrm{R}_{2} / \mathrm{R}_{1}=2.3 \pm 0.40, n=6 ; p=$ 0.03 , paired $t$ test), attributable to reduced presynaptic inhibition (Matsui et al., 2001; Sagdullaev et al., 2006). By contrast, for long ISIs, the time courses of the second and initial responses were similar (Fig. $6 A b, B$ ) (half-width ratio $\mathrm{R}_{2} / \mathrm{R}_{1}=$ $0.98 \pm 0.20, n=6 ; p=0.23$, paired $t$ test), suggesting that presynaptic inhibition similarly limited both responses. Thus, for short, but not longer, ISIs, presynaptic inhibition reduces $\mathrm{Pr}$, leading to reduced depression of the NMDAR component. This is in agreement with our findings that presynaptic inhibition from amacrine cells to BCs was depressed at short ISIs (Fig. 4). The time course of the AMPAR component (Fig. 6B,C) did not depend on ISI, indicating that it was unaffected by presynaptic inhibition, as shown in Figure 3.

\section{Discussion}

In this study, we use a retinal circuit to investigate how the dynamic interactions between excitation and presynaptic inhibition shape sensory signaling. While earlier work demonstrates that static in-

teractions between excitation and inhibition shape retinal output signals (Dong and Werblin, 1998; Matsui et al., 2001; Sagdullaev et al., 2006), our findings suggest that dynamic interactions also exist. This active interplay between excitation and presynaptic inhibition may enrich visual processing by enhancing the retinal response to closely spaced temporal events. Without this interplay, processing would be limited by synaptic depression (Singer and Diamond, 2006). Presynaptic inhibition has been shown to reduce synaptic depression within the rod-signaling pathway, presumably by truncating the light-induced depolarization of the rod bipolar synaptic terminal (Dunn and Rieke, 2008); however, its contribution to cone-mediated signals remained unknown (Demb and von Gersdorff, 2008). Here we show that presynaptic inhibition to cone BCs limits synaptic depression of NMDARmediated inputs to GCs. In turn, this could enhance novel visual signals and may underlie the psychophysical phenomenon of preferential detection of dynamic objects compared with static objects (Driver and Baylis, 1989; Vuong et al., 2006).

\section{Paired-pulse depression and gain control by presynaptic inhibition}

At many synapses, presynaptic inhibition reduces depression by limiting transmitter release, and preventing depletion of vesicles and/or saturation/desensitization of postsynaptic receptors (Wu and Saggau, 1997). We found that activation of presynaptic $\mathrm{GABA}_{C} \mathrm{Rs}$ limited release and reduced depression of the retinal output, but in a frequency-dependent manner (Fig. 2D). As the ISI shortened, the inhibitory effect exerted on BC output progressively decreased for the second response. As a result of this dynamic interplay, synaptic depression was progressively reduced. We found that this dynamic interaction was mediated by a frequency-dependent modulation of GC inputs, mediated exclusively by NMDARs, but not AMPARs.

The dynamic nature of synaptic depression that we observed represents interplay between excitatory and inhibitory mechanisms. Our results suggest that nonlinear interaction between presynaptic inhibition and excitatory transmission exists in the inner retina to strengthen its output by limiting depression for both electrically and light-evoked responses. This can be seen best by comparing the paired-pulse depression plots obtained with and without $\mathrm{GABA}_{\mathrm{C}} \mathrm{R}$-mediated presynaptic inhibition (Fig. $2 D)$. For both electrical and light stimuli, presynaptic inhibition reduced paired-pulse depression at short ISIs. Dynamic interactions between excitation and inhibition also occur in other parts of the CNS. Galarreta and Hestrin (1998) show in cortex that inhibitory synapses show weaker depression than excitatory synapses, stabilizing cortical firing. Klyachko and Stevens (2006) demonstrated that hippocampal excitatory and inhibitory synapses interact synergistically as an adaptive filter for natural spike trains. Our results demonstrate that similar interactions occur in the retina and may amplify responses to novel visual stimuli.

\section{Presynaptic inhibition differentially controls gain of AMPAR and NMDAR activation}

The inner retina is unique because presynaptic inhibition by $\mathrm{GABA}_{\mathrm{C}}$ Rs differentially affects AMPAR- and NMDAR-mediated components of GC eEPSCs (Matsui et al., 2001; Sagdullaev et al., 2006). These distinct effects are attributed to the spatial segregation of synaptic AMPARs and perisynaptic NMDARs (Chen and Diamond, 2002). Reducing presynaptic inhibition increased transmitter release, leading to stronger paired-pulse depression for both AMPAR- and NMDAR-mediated inputs to ON GCs 
(Fig. 1D,E). The similarities in depression for the AMPAR and NMDAR components, at short ISIs, suggested that AMPAR desensitization (Trussell et al., 1993; Brenowitz et al., 1998) did not contribute significantly to depression at BC synapses, in agreement with previous studies (Singer and Diamond, 2006; Dunn and Rieke, 2008). At the calyx of Held, Wong et al. (2003) determined that postsynaptic AMPAR desensitization does not contribute to synaptic depression at stimulus frequencies of $<10-30$ $\mathrm{Hz}$. We found that $\mathrm{GABA}_{\mathrm{C}} \mathrm{R}$-mediated presynaptic inhibition most prominently reduced depression over a low range of stimulus frequencies $(3-10 \mathrm{~Hz})$. Since responses were dominated by NMDARs (Fig. 3B), postsynaptic AMPAR desensitization, even if it did occur, would not significantly shape responses. Further, the time course of the AMPAR EPSCs was not altered when transmitter release was varied (Fig. 5B). Together, these observations suggest that depression was not attributable to postsynaptic AMPARs.

When $\mathrm{GABA}_{\mathrm{C}} \mathrm{R}$-mediated presynaptic inhibition limited transmitter release, it prevented the depression of AMPAR- and NMDAR-mediated GC responses. The effects of presynaptic inhibition on NMDARs, but not AMPARs, were frequency dependent. We previously showed that presynaptic inhibition, mediated by $\mathrm{GABA}_{\mathrm{C}} \mathrm{Rs}$, selectively controlled the spillover activation of perisynaptic NMDARs to extend the dynamic range of the retinal output (Sagdullaev et al., 2006). We attribute frequency-dependent differences to presynaptic inhibition selectively limiting the activation of postsynaptic NMDARs (Matsui et al., 2001; Sagdullaev et al., 2006). The present study reveals that the dynamic adjustments of excitatory transmission and presynaptic inhibition results in frequency-dependent modulation of NMDAR activation and nonlinear gain control of the retinal output. What causes this frequency-dependent interplay between excitation and presynaptic inhibition that result in dynamic modulation of NMDAR-mediated inputs to ON GCs?

Figure 7 illustrates a scheme that explains the dynamic interaction between excitation and presynaptic inhibition. For short ISIs, the excitatory output is depressed, presumably attributable to presynaptic depletion of vesicles. We showed that inhibition of the BC terminal also exhibits depression (Fig. 4), in agreement with a previous study ( $\mathrm{Li}$ et al., 2007). Because presynaptic inhibition is depressed at short ISIs, this suggests that reduced inhibition does not significantly suppress subsequent release, resulting in a larger response to the second stimulus. Alternatively, because the presynaptic inhibitory synapse is in series with the excitatory synapse, it would receive a weaker drive during the second stimulus, causing even less presynaptic inhibition. These two mechanisms are not mutually exclusive, and both could explain our data. Further studies that directly measure the inhibitory inputs to presynaptic terminal will help to dissect the precise mechanisms.

It is unlikely that serial inhibition across the amacrine cell network plays a major role in the reduction of depression by a presynaptic inhibition. Both light-evoked and electrically evoked short-term depression (STD) profiles were similar in the presence or absence of serial inhibition, in agreement with our earlier findings of presynaptic inhibition modulating glutamate release from BCs and shaping the retinal output (Sagdullaev et al., 2006).

\section{Physiological significance of frequency-dependent gain control of NMDAR activation}

What is the physiological role for dynamic interactions between reciprocal synapses that determine the retinal output? The mechanisms by which synaptic depression contributes to response gain control have been described for the retinal rod-signaling pathways (Dunn et al., 2006; Dunn and Rieke, 2008). Two sites of gain control have been identified that enhance the dynamic range of the retinal output; one is located within the photoreceptors and the second in the rod bipolar cell axon terminal (Dunn and Rieke, 2006; Dunn et al., 2006). Here we show that cone bipolar cell terminals control the gain of transmission to GCs and presynaptic inhibition limits the extent of depression at this synapse. Similar interactions have been observed in the inner retinal circuitry mediating rod-driven signals, where elimination of inhibition has exacerbated paired-pulse depression (Dunn and Rieke, 2008). We show in the cone pathway that this gain control mechanism is primarily mediated by NMDARs, which is likely due to the fact that strong cone-driven signals may result in spillover activation of perisynaptic NMDARs. In the cone-signaling pathway, presynaptic inhibition had additional effects. We found that nonlinear control of excitatory inputs to GCs by presynaptic inhibition contributes to gain control of retinal output by enhancing the responses during repetitive stimulation. Furthermore, our data suggest that adjusting the relative amounts of inhibition and excitation not only increases the response gain but might also enhance specific temporal features of visual input. Indeed, the most pronounced effect of presynaptic inhibition on pairedpulse depression was observed at ISIs shorter than $300 \mathrm{~ms}$, which falls within the temporal integration window set by saccade eye movements occurring every 100-500 ms (Sakatani and Isa, 2007).

It remains to be seen to what extent these mechanisms vary among the subtypes of ON GC populations found in the mouse retina (Sun et al., 2002; Badea and Nathans, 2004; Kong et al., 2005). Our earlier data suggest that excitatory input to all ON GC classes is modulated by a presynaptic inhibitory signal that is mediated by $\mathrm{GABA}_{\mathrm{C}}$ Rs (Sagdullaev et al., 2006). In this study, we similarly found that multiple ON GC classes display characteristic modulation by presynaptic inhibition when inputs are adjusted to an appropriate level. Hence, it is more likely that the occurrence of this phenomenon depends on the state of the synapse rather than particular ON GC types.

Our observations in the retina may represent the case of more general synaptic interactions that also occur in other sensory systems, such as synapses between mitral cells and granule interneurons of olfactory bulb (Mori and Shepherd, 1979; Jahr and Nicoll, 1980), and end-bulb synapses of auditory nerve (Otis and Trussell, 1996) and nucleus magnocellularis (Cook et al., 2003). Since feedback loops are essential elements of many neural circuits, our findings start to address more complex interactions that modulate the information processing throughout the CNS.

\section{References}

Abbott LF, Varela JA, Sen K, Nelson SB (1997) Synaptic depression and cortical gain control. Science 275:220-224.

Badea TC, Nathans J (2004) Quantitative analysis of neuronal morphologies in the mouse retina visualized by using a genetically directed reporter. J Comp Neurol 480:331-351.

Bellingham MC, Walmsley B (1999) A novel presynaptic inhibitory mechanism underlies paired pulse depression at a fast central synapse. Neuron 23:159-170.

Brenowitz S, David J, Trussell L (1998) Enhancement of synaptic efficacy by presynaptic GABA(B) receptors. Neuron 20:135-141.

Brenowitz S, Trussell LO (2001) Minimizing synaptic depression by control of release probability. J Neurosci 21:1857-1867.

Chen C, Regehr WG (2003) Presynaptic modulation of the retinogeniculate synapse. J Neurosci 23:3130-3135.

Chen C, Blitz DM, Regehr WG (2002) Contributions of receptor desensiti- 
zation and saturation to plasticity at the retinogeniculate synapse. Neuron 33:779-788.

Chen S, Diamond JS (2002) Synaptically released glutamate activates extrasynaptic NMDA receptors on cells in the ganglion cell layer of the rat retina. J Neurosci 22:2165-2173.

Cohen ED (2000) Light-evoked excitatory synaptic currents of X-type retinal ganglion cells. J Neurophysiol 83:3217-3229.

Cook DL, Schwindt PC, Grande LA, Spain WJ (2003) Synaptic depression in the localization of sound. Nature 421:66-70.

del Castillo J, Katz B (1954) Quantal components of the end-plate potential. J Physiol 124:560-573.

Demb JB, von Gersdorff H (2008) Ultraweak signals can cause synaptic depression and adaptation. Neuron 57:802-804.

Dittman JS, Regehr WG (1998) Calcium dependence and recovery kinetics of presynaptic depression at the climbing fiber to Purkinje cell synapse. J Neurosci 18:6147-6162.

Dong CJ, Werblin FS (1998) Temporal contrast enhancement via GABA $_{\mathrm{C}}$ feedback at bipolar terminals in the tiger salamander retina. J Neurophysiol 79:2171-2180.

Driver J, Baylis GC (1989) Movement and visual attention: the spotlight metaphor breaks down. J Exp Psychol Hum Percept Perform 15:448-456.

Dunn FA, Rieke F (2008) Single-photon absorptions evoke synaptic depression in the retina to extend the operational range of rod vision. Neuron 57:894-904.

Dunn FA, Doan T, Sampath AP, Rieke F (2006) Controlling the gain of rod-mediated signals in the mammalian retina. J Neurosci 26:3959-3970.

Dunn FA, Rieke F (2006) The impact of photoreceptor noise on retinal gain controls. Curr Opin Neurobiol 16:363-370.

Eggers ED, Lukasiewicz PD (2006a) GABAA, GABAC and glycine receptormediated inhibition differentially affects light-evoked signaling from mouse retinal rod bipolar cells. J Physiol 572:215-225.

Eggers ED, Lukasiewicz PD (2006b) Receptor and transmitter release properties set the time course of retinal inhibition. J Neurosci 26:9413-9425.

Euler T, Masland RH (2000) Light-evoked responses of bipolar cells in mammalian retina. J Neurophysiol 83:1817-1829.

Furukawa T, Matsuura S (1978) Adaptive rundown of excitatory postsynaptic potentials at synapses between hair cells and eight nerve fibres in the goldfish. J Physiol 276:193-209.

Galarreta M, Hestrin S (1998) Frequency-dependent synaptic depression and the balance of excitation and inhibition in the neocortex. Nat Neurosci 1:587-594.

Ghosh KK, Bujan S, Haverkamp S, Feigenspan A, Wässle H (2004) Types of bipolar cells in the mouse retina. J Comp Neurol 469:70-82.

Higgs MH, Lukasiewicz PD (1999) Glutamate uptake limits synaptic excitation of retinal ganglion cells. J Neurosci 19:3691-3700.

Jahr CE, Nicoll RA (1980) Dendrodendritic inhibition: demonstration with intracellular recording. Science 207:1473-1475.

Klyachko VA, Stevens CF (2006) Excitatory and feed-forward inhibitory hippocampal synapses work synergistically as an adaptive filter of natural spike trains. PLoS Biol 4:e207.

Kong JH, Fish DR, Rockhill RL, Masland RH (2005) Diversity of ganglion cells in the mouse retina: unsupervised morphological classification and its limits. J Comp Neurol 489:293-310.

Li GL, Vigh J, von Gersdorff H (2007) Short-term depression at the reciprocal synapses between a retinal bipolar cell terminal and amacrine cells. J Neurosci 27:7377-7385.

Lukasiewicz PD, Roeder RC (1995) Evidence for glycine modulation of excitatory synaptic inputs to retinal ganglion cells. J Neurosci 15:45924601.

Matsui K, Hasegawa J, Tachibana M (2001) Modulation of excitatory synaptic transmission by $\mathrm{GABA}(\mathrm{C})$ receptor-mediated feedback in the mouse inner retina. J Neurophysiol 86:2285-2298.

McCall MA, Lukasiewicz PD, Gregg RG, Peachey NS (2002) Elimination of the rhol subunit abolishes $\mathrm{GABA}_{\mathrm{C}}$ receptor expression and alters visual processing in the mouse retina. J Neurosci 22:4163-4174.

McMahon MJ, Packer OS, Dacey DM (2004) The classical receptive field surround of primate parasol ganglion cells is mediated primarily by a non-GABAergic pathway. J Neurosci 24:3736-3745.

Mori K, Shepherd GM (1979) Synaptic excitation and long-lasting inhibition of mitral cells in the in vitro turtle olfactory bulb. Brain Res 172:155-159.

Nowak L, Bregestovski P, Ascher P, Herbet A, Prochiantz A (1984) Magnesium gates glutamate-activated channels in mouse central neurones. Nature 307:462-465.

O'Donovan MJ, Rinzel J (1997) Synaptic depression: a dynamic regulator of synaptic communication with varied functional roles. Trends Neurosci 20:431-433.

Otis TS, Trussell LO (1996) Inhibition of transmitter release shortens the duration of the excitatory synaptic current at a calyceal synapse. J Neurophysiol 76:3584-3588.

Pang JJ, Gao F, Wu SM (2003) Light-evoked excitatory and inhibitory synaptic inputs to ON and OFF alpha ganglion cells in the mouse retina. J Neurosci 23:6063-6073.

Peichl L, Wässle H (1981) Morphological identification of on- and offcentre brisk transient $(\mathrm{Y})$ cells in the cat retina. Proc R Soc Lond B Biol Sci 212:139-153.

Rabl K, Cadetti L, Thoreson WB (2006) Paired-pulse depression at photoreceptor synapses. J Neurosci 26:2555-2563.

Sagdullaev BT, McCall MA, Lukasiewicz PD (2006) Presynaptic inhibition modulates spillover, creating distinct dynamic response ranges of sensory output. Neuron 50:923-935

Sakatani T, Isa T (2007) Quantitative analysis of spontaneous saccade-like rapid eye movements in C57BL/6 mice. Neurosci Res 58:324-331.

Singer JH, Diamond JS (2006) Vesicle depletion and synaptic depression at a mammalian ribbon synapse. J Neurophysiol 95:3191-3198.

Sun W, LiN, He S (2002) Large-scale morphological survey of mouse retinal ganglion cells. J Comp Neurol 451:115-126.

Trussell LO, Fischbach GD (1989) Glutamate receptor desensitization and its role in synaptic transmission. Neuron 3:209-218.

Trussell LO, Thio LL, Zorumski CF, Fischbach GD (1988) Rapid desensitization of glutamate receptors in vertebrate central neurons. Proc Natl Acad Sci U S A 85:2834-2838.

Trussell LO, Zhang S, Raman IM (1993) Desensitization of AMPA receptors upon multiquantal release. Neuron 10:1185-1196.

Tsodyks MV, Markram H (1997) The neural code between neocortical pyramidal neurons depends on neurotransmitter release probability. Proc Natl Acad Sci U S A 94:719-723.

Verweij J, Kamermans M, Spekreijse H (1996) Horizontal cells feed back to cones by shifting the cone calcium-current activation range. Vis Res 36:3943-3953.

Völgyi B, Abrams J, Paul DL, Bloomfield SA (2005) Morphology and tracer coupling pattern of alpha ganglion cells in the mouse retina. J Comp Neurol 492:66-77.

von Gersdorff H, Matthews G (1997) Depletion and replenishment of vesicle pools at a ribbon-type synaptic terminal. J Neurosci 17:1919-1927.

Vuong QC, Hof AF, Bülthoff HH, Thornton IM (2006) An advantage for detecting dynamic targets in natural scenes. J Vis 6:87-96.

Wong AY, Graham BP, Billups B, Forsythe ID (2003) Distinguishing between presynaptic and postsynaptic mechanisms of short-term depression during action potential trains. J Neurosci 23:4868-4877.

Wu LG, Saggau P (1997) Presynaptic inhibition of elicited neurotransmitter release. Trends Neurosci 20:204-212.

Zhang J, Slaughter MM (1995) Preferential suppression of the on pathway by $\mathrm{GABA}_{\mathrm{C}}$ receptors in the amphibian retina. J Neurophysiol 74:15831592.

Zucker RS, Regehr WG (2002) Short-term synaptic plasticity. Annu Rev Physiol 64:355-405. 